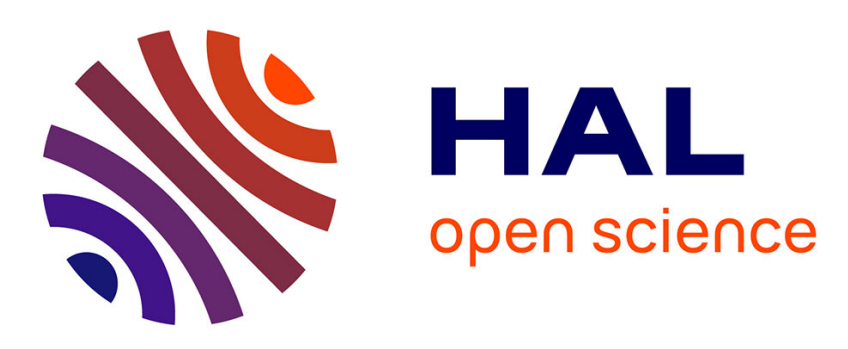

\title{
Ab initio study of electronic temperature effects on magnetic materials properties
}

Philippe Scheid, Grégory Malinowski, Stéphane Mangin, Sébastien Lebègue

\section{To cite this version:}

Philippe Scheid, Grégory Malinowski, Stéphane Mangin, Sébastien Lebègue. Ab initio study of electronic temperature effects on magnetic materials properties. Physical Review B: Condensed Matter and Materials Physics (1998-2015), 2019, 99 (17), 10.1103/PhysRevB.99.174415 . hal-02185986

\section{HAL Id: hal-02185986 \\ https://hal.univ-lorraine.fr/hal-02185986}

Submitted on 17 Jul 2019

HAL is a multi-disciplinary open access archive for the deposit and dissemination of scientific research documents, whether they are published or not. The documents may come from teaching and research institutions in France or abroad, or from public or private research centers.
L'archive ouverte pluridisciplinaire HAL, est destinée au dépôt et à la diffusion de documents scientifiques de niveau recherche, publiés ou non, émanant des établissements d'enseignement et de recherche français ou étrangers, des laboratoires publics ou privés.

$$
\text { Copyright }
$$




\title{
$A b$ initio study of electronic temperature effects on magnetic materials properties
}

\author{
Philippe Scheid, ${ }^{1,2, *}$ Gregory Malinowski, ${ }^{2}$ Stéphane Mangin, ${ }^{2}$ and Sébastien Lebègue ${ }^{1}$ \\ ${ }^{1}$ Université de Lorraine, LPCT, CNRS, UMR 7019, Boîte Postale 70239, 54506 Vandoeuvre-lès-Nancy Cedex, France \\ ${ }^{2}$ Université de Lorraine, IJL, CNRS, UMR 7198, Boîte Postale 70239, 54000 Nancy Cedex, France
}

(Received 21 December 2018; revised manuscript received 18 March 2019; published 17 May 2019)

\begin{abstract}
We present $a b$ initio calculations of the electronic temperature dependence of the magnetization, electronic energy, and specific heat of FePt $L 1_{0}$, fcc-Ni, hcp-Co, and bcc-Fe. We find that atomic magnetic moments in each compound disappear at very high temperature, ranging from 3100 to $8100 \mathrm{~K}$. However, for some compounds, the consequences of this phenomenon are noticeable on the electronic energy and specific heat even at low electronic temperature. Consequently, large deviations from the Sommerfeld approximation and from some previous work that did not take into account explicitly the dependence of the electronic structure on the electronic temperature are shown. Our results are of interest in the field of laser-induced ultrafast magnetization dynamics, since they provide a more precise estimate of the electronic specific heat that enters in the three-temperature model.
\end{abstract}

DOI: 10.1103/PhysRevB.99.174415

\section{INTRODUCTION}

Interaction of ultrafast and intense light pulses with magnetic materials has led to the discovery of many intriguing phenomena, such as ultrafast demagnetization [1] and alloptical switching [2-4]. Our understanding of these experimental facts is tightly bound to our knowledge of the properties of materials when the electronic temperature is raised far above room temperature by the laser. Indeed, by using such femtosecond light pulses, it is possible to bring matter in previously unreachable states where the electronic temperature, $T_{e}$, can be well above the phonon temperature [5,6]. While the mechanisms involved in the ultrafast demagnetization and all-optical switching are still heavily debated, our current rationalization of these experimental facts relies mostly on the two- or three-temperature model and, consequently, on our knowledge of the specific heat of the different baths.

To assess the electronic specific heat, the Sommerfeld approximation is usually chosen [5,6]. However, this approximation is only meaningful at low $T_{e}$, and one may question its domain of validity. Lin et al. [7] went beyond this approximation for a wide range of magnetic and nonmagnetic compounds and found good agreement between both methods up to several thousand Kelvins, while neglecting the effect of $T_{e}$ on the electronic structure.

In this work, we use $a b$ initio density functional theory (DFT) to compute the static dependence on $T_{e}$ of different electronic properties for fcc-Ni, hcp-Co, bcc-Fe, and FePt $L 1_{0}$. Specifically, we self-consistently compute the dependence of the magnetization on $T_{e}$. By doing so, we define a temperature, $T_{S}$, where $S$ refers to Stoner, at which the atomic magnetic moments completely disappear. This phenomenon has been mentioned in the literature, in particular by Gunnarsson [8], but its implications have never been fully explored. Therefore, we compute the electronic energy and specific heat

*philippe.scheid@univ-lorraine.fr and show the presence of a strong signature of the Stoner spin-flip process, missing from previous work [7] and from the Sommerfeld approximation.

This paper is organized as follows. In Sec. II we briefly summarize how Gunnarsson computed the Stoner temperature and how we build upon his results by using modern $a b$ initio DFT tools. Using this framework, we explain how we compute the electronic energy and specific heat and how it contrasts with what has been done previously. In Sec. III we present and discuss our results for the Stoner temperature, the electronic energy, and the specific heat, and we compare them with previous works. Also, we discuss our results in the context of the previously mentioned field of light-induced ultrafast dynamics. Finally, in Sec. IV we present our conclusions and outlooks.

\section{THEORY}

\section{A. Self-consistent calculation of electron-dependent magnetic properties}

Gunnarsson [8] was the first to use DFT to reproduce the experimental atomic magnetic moment of bcc-Fe, hcp-Co, and fcc-Ni and to assess the behavior of magnetization when $T_{e}$ changes. As spin-polarized calculations were not available at this time, he computed the magnetization by including the spin difference in the exchange correlation potential in the local spin-density approximation as a first-order perturbation. By solving $I\left(\epsilon_{F}\right) \int_{-\infty}^{\infty} \frac{\partial f\left(\epsilon, \mu, T_{e}\right)}{\partial \epsilon} g(\epsilon) d \epsilon+1=0$, where $I(\epsilon)$ is the Stoner parameter, $f$ is the Fermi-Dirac distribution, $\mu$ is the chemical potential, and $g(\epsilon)$ is the density of states (DOS), he assessed the critical electronic temperature, $T_{e}$, at which the atomic magnetic moments would disappear and found values well above $T_{C}$, the Curie temperature of each compound. He explained this discrepancy by pointing out that even though band theory can describe the formation of atomic magnetic moments and their longitudinal temperature dependence, the Curie temperature should, however, be 
influenced by the direct or indirect exchange interactions between atomic moments, as exemplified by the Heisenberg model. As the temperature increases, so do the transversal fluctuations, and the global magnetization is on average suppressed, even though atomic moments still exist. To distinguish both effects, we define $T_{e}$ at which the atomic moments disappear as the Stoner temperature $T_{S}$.

Nowadays, spin-polarized calculations are common practice, and they have shown great success in reproducing the local atomic moment values. Moreover, the possibility of performing DFT calculations at finite electronic temperature [9] allows us to build on Gunnarsson's calculation of $T_{S}$ by performing self-consistent $a b$ initio computations for a wide range of $T_{e}$.

\section{B. Self-consistent calculation of electronic energy and specific heat}

In metals, changes in the electronic energy due to $T_{e}$ are commonly dealt with by using the Sommerfeld approximation. In this framework, the electron specific heat, $C_{e}$, depends linearly on $T_{e}$ and on $g\left(\epsilon_{F}\right)$, the DOS at the Fermi energy, such as $C_{e}=\gamma T$ with $\gamma=\frac{\pi^{2}}{3} k_{B}^{2} g\left(\epsilon_{F}\right)$. However, this approximation is only valid at low electronic temperature, at which the Fermi-Dirac distribution is close to the Heaviside function. Recently, Lin et al. [7] went beyond this approximation and fully took into account the DOS shape in their calculation. Using DFT, they computed the DOS of different compounds at low $T_{e}$, from which they accessed the temperature-dependent electronic energy using

$$
E_{e}\left(T_{e}\right)=\int_{-\infty}^{\infty} f\left(\epsilon, \mu, T_{e}\right) g(\epsilon) \epsilon d \epsilon,
$$

where $E_{e}$ is the electronic energy and $\mu$ is accessed by solving the implicit equation

$$
N_{e}=\int_{-\infty}^{\infty} f\left(\epsilon, \mu, T_{e}\right) g(\epsilon) d \epsilon,
$$

where $N_{e}$ is the number of electrons in the unit cell.

Finally, they obtained the electronic specific heat with

$$
C_{e}\left(T_{e}\right)=\frac{\partial E_{e}\left(T_{e}\right)}{\partial T_{e}} .
$$

In their work, Lin et al. [7] considered the density of states to be independent of $T_{e}$. However, in light of the work of Gunnarsson [8], one may ask how the existence of a Stoner temperature influences their results when dealing with magnetic materials.

In contrast with Lin et al. [7], we will compute the DOS for a wide range of $T_{e}$. Therefore, we can rewrite Eq. (1) as

$$
E_{e}\left(T_{e}\right)=\int_{-\infty}^{\infty} f\left(\epsilon, \mu, T_{e}\right) g\left(\epsilon, T_{e}\right) \epsilon d \epsilon
$$

and Eq. (2) as

$$
N_{e}=\int_{-\infty}^{\infty} f\left(\epsilon, \mu, T_{e}\right) g\left(\epsilon, T_{e}\right) d \epsilon .
$$

\section{Computational details}

To solve the spin-polarized Kohn-Sham equations [10,11], we use the VASP package [12-14] with projector augmented wave potentials $[15,16]$ and the generalized gradient approximation [17]. The energy cutoff of the plane-wave basis is set to $500 \mathrm{eV}$. Calculations are considered to be converged when the difference in energy between two electronic loops is lower than $10^{-8} \mathrm{eV}$. The Fermi-Dirac distribution is used to selfconsistently compute the electronic ground state for $100<$ $T_{e}<13000 \mathrm{~K}$, sampled with 120 points. We use a $k$-point grid of $36 \times 36 \times 36$ or equivalent for noncubic geometries, except at low $T_{e}$, where we increase it to $56 \times 56 \times 56$ to ensure convergence. Then the DOSs are obtained from the previous calculation using the tetrahedron method with Blöchl corrections [18].

\section{RESULTS AND DISCUSSION}

\section{A. Dependence of the electronic structure on the electronic temperature}

The $T_{e}$ dependence of the electronic structure is evidenced by Fig. 1, showing how the self-consistently calculated spinpolarized DOS evolves with $T_{e}$. At low $T_{e}$ we find that, as expected, all the compounds studied here are magnetic with $0.6 \mu_{B} /$ at. for fcc-Ni, $1.6 \mu_{B} /$ at. for hcp-Co, $2.2 \mu_{B} /$ at. for bcc-Fe, and [3.1, 0.3] $\mu_{B}$ for $[\mathrm{Fe}, \mathrm{Pt}]$ in FePt $L 1_{0}$. As a consequence, the minority- and majority-spin DOSs are not identical in the lower range of $T_{e}$, and the integrated difference gives the magnetization. As $T_{e}$ increases, the majority- and minority-spin DOSs progressively evolve until they become identical at the critical Stoner temperature, $T_{S}$. This underscores the effect of $T_{e}$ on the electron eigenstates, which has been ignored so far in this context [7].

Figure 2 shows the magnetization per unit cell as a function of $T_{e}$ for each studied compound. The behavior is typical of a ferromagnetic-paramagnetic (as defined by Pauli) phase transition. Table I summarizes the Stoner temperature, ranging from $3100 \mathrm{~K}$ in the case of fcc-Ni up to $8100 \mathrm{~K}$ in the case of FePt $L 1_{0}$, as well as the values calculated by Gunnarsson [8] when available. Our computed values are systematically larger than those of [8]. This difference may be due to the assumption of the temperature independence of the Stoner parameter as well as to his first-order perturbation treatment of the exchange splitting. As pointed out by Gunnarsson, $T_{S}$ is not directly correlated to $T_{C}$ since $T_{S}$ is related to the intra-atomic exchange giving rise to atomic magnetic moments, while $T_{C}$ is mainly a consequence of the interatomic direct and/or indirect exchange. However, as $T_{S}$ is related to the local exchange, a larger atomic moment induces a larger $T_{S}$.

To explain such a behavior, one may recall that magnetism is a direct consequence of the exchange interaction between electrons, which is a quantum effect arising from the antisymmetric nature of their wave functions and the Coulomb repulsion. This interaction tends to align electron spins, and in this way it favors magnetic states. However, the penalty resulting from the increase of the kinetic and potential energy counteracts this magnetized state, and in most cases completely cancels it. Nevertheless, when the Fermi 


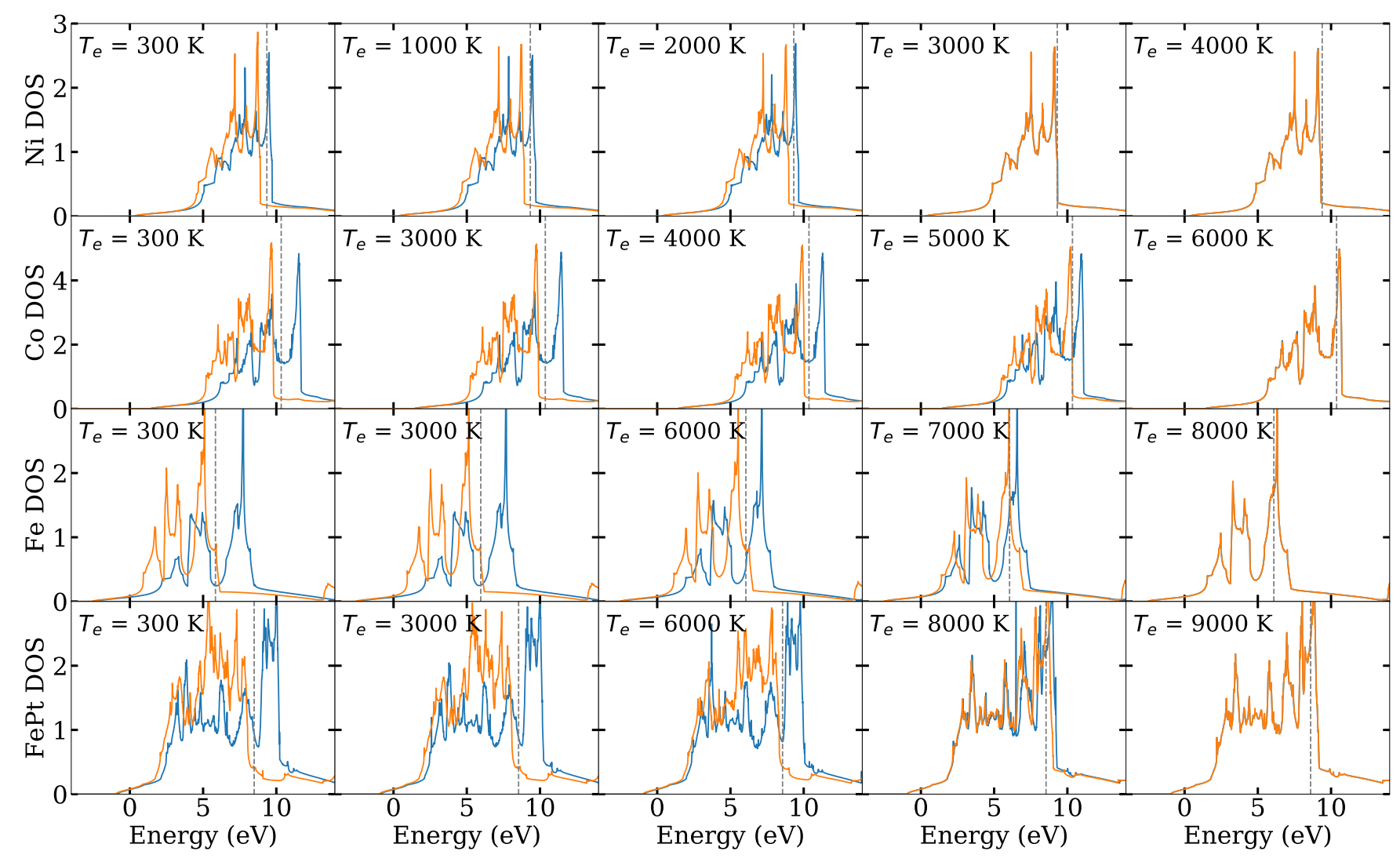

FIG. 1. Spin-polarized density of states of fcc-Ni, hcp-Co, bcc-Fe, and FePt $L 1_{0}$ for different values of the electronic temperature chosen below and above the Stoner temperature. The majority- (minority-) spin DOSs are in orange (blue), and the value of $\mu$, the chemical potential, is indicated by a vertical dashed line.

energy lies within a peak of the DOS, as is the case for all the compounds studied here, the increase of the kinetic energy resulting from spin flips is greatly reduced. These considerations are summarized by the well-known Stoner criterion: $\operatorname{Ig}\left(\epsilon_{F}\right)>1$, where $I$ is the Stoner parameter $[19,20]$. However, as $T_{e}$ increases, electrons start to occupy higherenergy states. When $T_{e}$ becomes high enough, the smearing of the Fermi-Dirac distribution allows the minority-spin peak to start being occupied again. Consequently, the number of electrons with high kinetic energy increases. To prevent this, the compounds relax to a nonmagnetic state where the minority-

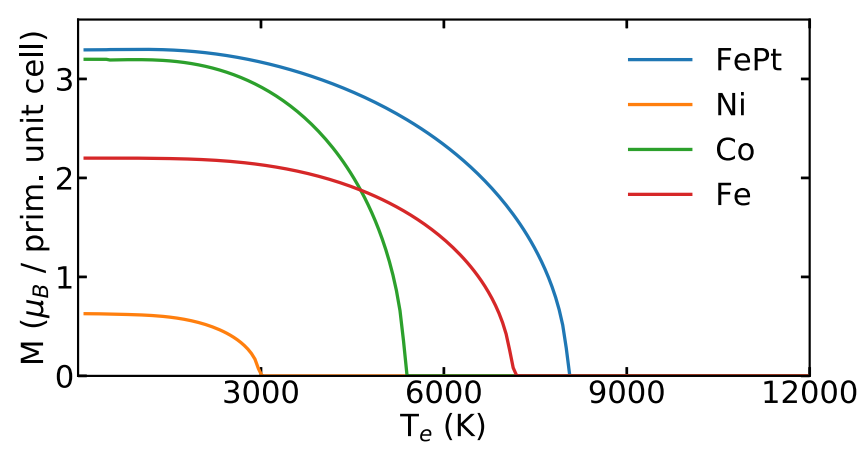

FIG. 2. Magnetic moment per unit cell against the electronic temperature. In every compound, we observe a loss of magnetization when the temperature is above $T_{S}$. spin DOS shifts down in energy and the majority-spin DOS shifts up.

This strong $T_{e}$ dependence of the electronic eigenstates, not taken into account in [7], raises the question of the $T_{e}$ dependence of other electronic properties, such as the electronic energy and specific heat.

\section{B. Consequences of the electronic temperature on the electronic specific heat}

Figure 3 shows the electronic energy as a function of $T_{e}$ calculated with the temperature-dependent DOS method (referred to as TDD) using Eq. (4) and with the temperature-

TABLE I. Calculated magnetic moment per unit cell and per atom and calculated Stoner temperature $T_{S}$ for all the compounds studied here. For comparison, we added the results from Gunnarsson [8]. In contrast with $T_{S}$, we also recall the experimental Curie temperature.

\begin{tabular}{lccccc}
\hline \hline & $\begin{array}{c}M \\
\left(\mu_{B} / \text { cell }\right)\end{array}$ & $M_{\text {at. }}\left(\mu_{B}\right)$ & $T_{S}(\mathrm{~K})$ & $\begin{array}{c}T_{S}(\mathrm{~K}) \\
\text { from [8] }\end{array}$ & $\begin{array}{r}T_{C}(\mathrm{~K}) \\
\text { (Expt.) }\end{array}$ \\
\hline FePt $L 1_{0}$ & 3.4 & $3.1,0.3$ & 8100 & & 750 \\
fcc-Ni & 0.6 & 0.6 & 3100 & 2900 & 627 \\
hcp-Co & 3.2 & 1.6 & 5500 & $3300-4800$ & 1400 \\
bcc-Fe & 2.2 & 2.2 & 7300 & $4400-6200$ & 1043 \\
\hline \hline
\end{tabular}



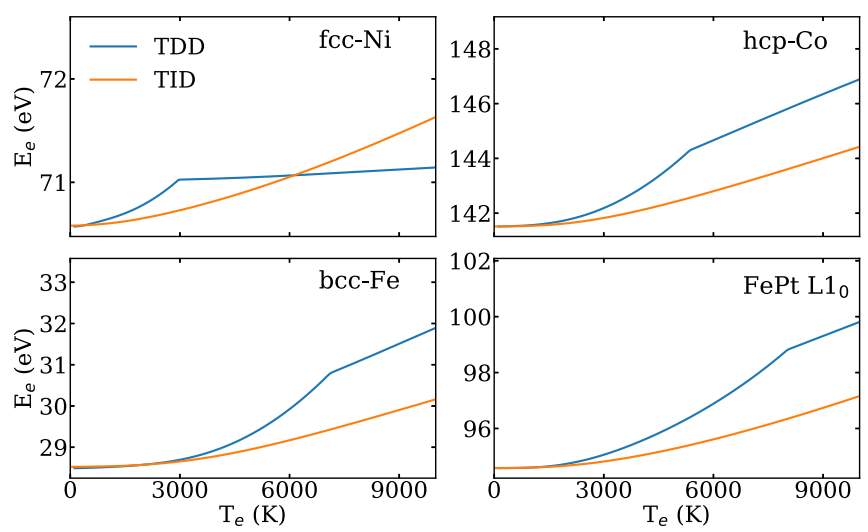

FIG. 3. Electronic energy per unit cell against the electronic temperature for fcc-Ni, hcp-Co, bcc-Fe, and FePt $L 1_{0}$. The electronic energy obtained from TDD (TID) calculations is in blue (orange).

independent DOS (TID) using Eq. (1). In the latter case, we use the DOS computed with a Fermi smearing of $0.01 \mathrm{eV}$, which induces a slight difference (less than $0.01 \mathrm{eV}$ ) with the TDD method when $T_{e} \rightarrow 0 \mathrm{~K}$. For every compound, the temperature dependences of TDD and TID curves are significantly different. At low $T_{e}$, the calculated energies are identical for both methods, as the effect of electronic temperature is negligible. As $T_{e}$ increases, the TDD electronic energies systematically become larger than the TID ones. However, the rate at which they differ as $T_{e}$ increases is different. In the case of fcc-Ni, both methods give a similar result only when $T_{e} \rightarrow 0 \mathrm{~K}$. For hcp-Co, bcc-Fe, and FePt $L 1_{0}$, the TDD and TID energies are similar for a wider range of $T_{e}$. Another striking feature of the TDD curves is the presence of a singularity at the Stoner temperature, at which the paraboliclike behavior, characteristic of the lower-temperature range, becomes quasilinear.

Figure 4 shows the electronic specific heat calculated using Eq. (3) and the TID and TDD approximations to compute the electronic energy. We compare our results with the specific heat obtained by using the Sommerfeld approximation, only valid at low temperature. The TID specific heat of Co reproduces that from Lin et al. [7]. The TID and the Sommerfeld heat capacities are always in agreement at low temperature, nevertheless it is not necessarily the case of the heat capacity calculated with the TDD method. Moreover, the TDD specific heats show a strong signature of the magnetic-paramagnetic second-order phase transition, as evidenced by the increase abruptly ending at $T_{S}$. This feature strongly differentiates the TID and TDD specific heat and highlights the necessity of taking explicitly into account the DOS temperature dependence.

At low $T_{e}$, in the case of bcc-Fe, $T_{S}$ is high enough that the influence of Stoner spin-flip processes on the eigenstates is negligible. Therefore, below $2000 \mathrm{~K}$, the TDD heat capacity matches with the Sommerfeld and TID heat capacity. In the case of fcc-Ni, which has the lowest $T_{S}$, Stoner spin-flip processes are noticeable at any temperature below $T_{S}$, such that the TID heat capacity, as well as the Sommerfeld heat capacity, deviates from the TDD specific heat. Finally, despite the larger magnetic moment carried by $\mathrm{Fe}$ in $\mathrm{FePt} L 1_{0}$ than in bcc-Fe, its TDD heat capacity differs from the TID and
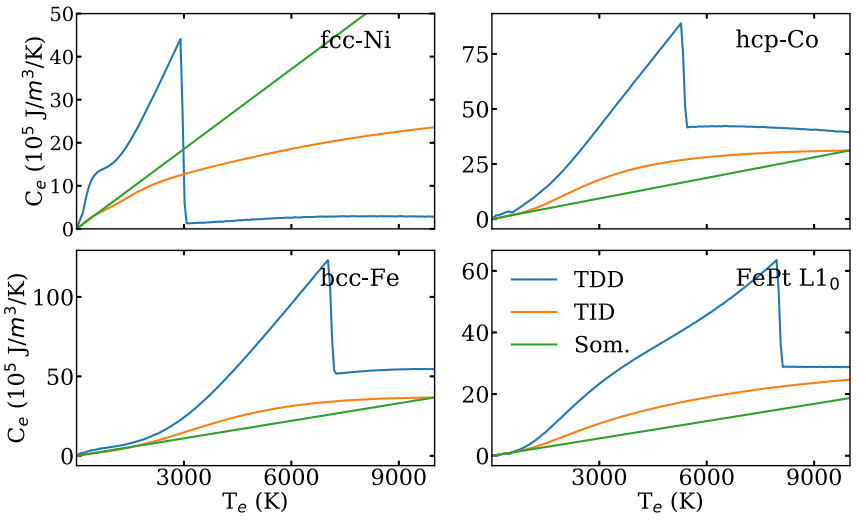

FIG. 4. Electronic specific heat vs the electronic temperature for fcc-Ni, hcp-Co, bcc-Fe, and FePt $L 1_{0}$ computed with different approximations.

the Sommerfeld heat capacity at lower temperature than in bcc-Fe. This discrepancy may be due to the presence of the Pt atom, carrying $0.3 \mu_{B}$, on which the effects of $T_{e}$ are more noticeable in the lower-temperature range. In Fig. 4 we notice that fcc-Ni not only stands out by its very low $T_{S}$ but also by its very small heat capacity above $T_{S}$ compared to the other compounds. Indeed, because of its $d$ shell occupied by nine electrons, and thus almost completely filled, the increase of $T_{e}$ shifts the chemical potential above the DOS peak caused by the $d$ shell, as seen in the fcc-Ni DOS (Fig. 1). This significantly smaller DOS at $\mu$ results in an equally smaller specific heat. Such a behavior is not seen for the other compounds, as $\mathrm{Co}$ and $\mathrm{Fe}$ have, respectively, seven and eight electrons on their $d$ shells. As a consequence, their chemical potentials still lie in the DOS peak when the electronic temperature is higher than $T_{S}$.

\section{About ultrafast magnetization dynamics}

Recent advances in the generation of ultrafast and intense laser pulses opened many new research fields. One of them is the study of the influence of such a light on magnetic materials. It has been shown that magnetization can be deterministically manipulated for a wide range of magnetic thin films [2-4] by using femtosecond light pulses. Moreover, the laser pulse also produces an ultrafast demagnetization happening on a $10-100 \mathrm{fs}$ timescale $[1,21,22]$. The mechanisms driving such a dynamics are still heavily debated. In the case of the ultrafast demagnetization phenomenon, the two main candidates are the production of magnons reducing the average magnetization, and the Stoner spin-flip processes reducing the atomic magnetization [5,21-24].

The Stoner temperature and its connection to the on-site exchange may provide valuable insights for the understanding of the ultrafast demagnetization. Indeed, in these experiments the laser energy is primarily absorbed by the electronic bath. Consequently, assuming that an electronic temperature can be defined shortly after the laser pulse, $T_{e}$ rises from the ambient temperature $T_{r}$ to well above $T_{C}$ while the phonon bath is still at $T_{r}$. This idealized state is what we reproduce by performing $a b$ initio DFT as we vary $T_{e}$ while considering the nucleus fixed, i.e., at $0 \mathrm{~K}$, as in the Born-Oppenheimer approximation. 
In this framework, a simple explanation of the ultrafast loss of magnetization could be that the electronic temperature exceeds $T_{S}$. Nevertheless, except in the case of fcc-Ni, reaching such temperatures would cause a melting of the compound. This failure to describe the ultrafast demagnetization may indicate that one has to take into account the dynamical aspect of light-matter interaction, and more specifically with the exchange splitting producing the atomic magnetization. This can be achieved using real-time TD-DFT [24].

However, our results show that the electronic temperature has a strong impact on the electronic specific heat, which is a key parameter in modeling the dynamics of electrons, phonons, and spins within the two- to three-temperature model [6]. The electronic specific heat is usually computed using the Sommerfeld approximation. As a consequence, it is strongly underestimated as soon as the electronic temperature increases. The present work provides more precise estimates of this key quantity, which could be taken as input parameters for future investigations.

\section{CONCLUSION}

The static dependence of the electronic properties of fcc$\mathrm{Ni}$, hcp-Co, bcc-Fe, and FePt $L 1_{0}$ was studied with DFT for a wide range of electronic temperature. In this framework, we were able to precisely compute the Stoner temperature, at which the intra-atomic exchange-splitting, and thus the atomic magnetic moment, disappears for all the compounds. We found values much higher than the Curie temperatures of the different compounds. Furthermore, these values are found to be correlated with the value of the largest atomic magnetic moment in the cell.

We demonstrated that this phenomenon has a strong signature on the electronic energy, and consequently on the specific heat. Such effects have been ignored in previous studies [5,7] and induce large deviations from the Sommerfeld approximation and the temperature-independent DOS approximation, even at relatively low electronic temperature.

These results evidence the fact that the Sommerfeld approximation is not sufficient to obtain a correct temperature dynamics in the two- to three-temperature model, commonly used to describe ultrafast and intense light-matter interactions. Finally, the Stoner temperatures we obtained are, except in the case of fcc-Ni, too high to be reached without destroying the compound. This suggests that dynamical effects [24-26] play a primary role in the process of ultrafast demagnetization. Furthermore, the specific-heat contributions of dynamical effects such as spin fluctuations have also been evidenced [27], and recent $a b$ initio studies showed their presence in $3 d$ metals [28,29].

\section{ACKNOWLEDGMENTS}

The authors are very grateful to Peter Oppeneer, Pablo Maldonado, and Marco Berritta for the many insightful and stimulating discussions. P.S. was supported by the French PIA project Lorraine Université d'Excellence, reference ANR-15IDEX-0004.
[1] E. Beaurepaire, J.-C. Merle, A. Daunois, and J.-Y. Bigot, Phys. Rev. Lett. 76, 4250 (1996).

[2] S. Mangin, M. Gottwald, C.-H. Lambert, D. Steil, V. Uhlî́, L. Pang, M. Hehn, S. Alebrand, M. Cinchetti, G. Malinowski, Y. Fainman, M. Aeschlimann, and E. E. Fullerton, Nat. Mater. 13, 286 (2014).

[3] C.-H. Lambert, S. Mangin, B. S. D. C. S. Varaprasad, Y. K. Takahashi, M. Hehn, M. Cinchetti, G. Malinowski, K. Hono, Y. Fainman, M. Aeschlimann, and E. E. Fullerton, Science 345 1337 (2014).

[4] I. Radu, K. Vahaplar, C. Stamm, T. Kachel, N. Pontius, H. A. Dürr, T. A. Ostler, J. Barker, R. F. L. Evans, R. W. Chantrell, A. Tsukamoto, A. Itoh, A. Kirilyuk, T. Rasing, and A. V. Kimel, Nature (London) 472, 205 (2011).

[5] B. Koopmans, G. Malinowski, F. Dalla Longa, D. Steiauf, M. Fähnle, T. Roth, M. Cinchetti, and M. Aeschlimann, Nat. Mater. 9, 259 (2010).

[6] J. Mendil, P. Nieves, O. Chubykalo-Fesenko, J. Walowski, T. Santos, S. Pisana, and M. Münzenberg, Sci. Rep. 4, 3980 (2014).

[7] Z. Lin, L. V. Zhigilei, and V. Celli, Phys. Rev. B 77, 075133 (2008).

[8] O. Gunnarsson, J. Phys. F 6, 587 (1976).

[9] N. D. Mermin, Phys. Rev. 137, A1441 (1965).

[10] P. Hohenberg and W. Kohn, Phys. Rev. 136, B864 (1964).

[11] W. Kohn and L. J. Sham, Phys. Rev. 140, A1133 (1965).
[12] G. Kresse and J. Hafner, Phys. Rev. B 47, 558 (1993).

[13] G. Kresse and J. Furthmüller, Phys. Rev. B 54, 11169 (1996).

[14] G. Kresse and J. Furthmüller, Comput. Mater. Sci. 6, 15 (1996).

[15] G. Kresse and D. Joubert, Phys. Rev. B 59, 1758 (1999).

[16] P. E. Blöchl, Phys. Rev. B 50, 17953 (1994).

[17] J. P. Perdew, K. Burke, and M. Ernzerhof, Phys. Rev. Lett. 77, 3865 (1996).

[18] P. E. Blöchl, O. Jepsen, and O. K. Andersen, Phys. Rev. B 49, 16223 (1994).

[19] M. P. Marder, Condensed Matter Physics (Wiley, Hoboken, New Jersey, 2010).

[20] W. Nolting and A. Ramakanth, Quantum Theory of Magnetism (Springer, Berlin, 2009).

[21] S. Eich, M. Plötzing, M. Rollinger, S. Emmerich, R. Adam, C. Chen, H. C. Kapteyn, M. M. Murnane, L. Plucinski, D. Steil, B. Stadtmüller, M. Cinchetti, M. Aeschlimann, C. M. Schneider, and S. Mathias, Sci. Adv. 3, e1602094 (2017).

[22] P. Tengdin, W. You, C. Chen, X. Shi, D. Zusin, Y. Zhang, C. Gentry, A. Blonsky, M. Keller, P. M. Oppeneer, H. C. Kapteyn, Z. Tao, and M. M. Murnane, Sci. Adv. 4, eaap9744 (2018).

[23] E. Turgut, D. Zusin, D. Legut, K. Carva, R. Knut, J. M. Shaw, C. Chen, Z. Tao, H. T. Nembach, T. J. Silva, S. Mathias, M. Aeschlimann, P. M. Oppeneer, H. C. Kapteyn, M. M. Murnane, and P. Grychtol, Phys. Rev. B 94, 220408(R) (2016). 
[24] K. Krieger, J. K. Dewhurst, P. Elliott, S. Sharma, and E. K. U. Gross, J. Chem. Theor. Comput. 11, 4870 (2015).

[25] K. Carva, M. Battiato, D. Legut, and P. M. Oppeneer, Phys. Rev. B 87, 184425 (2013).

[26] B. Y. Mueller, A. Baral, S. Vollmar, M. Cinchetti, M. Aeschlimann, H. C. Schneider, and B. Rethfeld, Phys. Rev. Lett. 111, 167204 (2013).
[27] D. J. Kim, New Perspectives in Magnetism of Metals (Springer, New York, 2013).

[28] A. L. Wysocki, A. Kutepov, and V. P. Antropov, Phys. Rev. B 94, 140405(R) (2016).

[29] A. L. Wysocki, V. N. Valmispild, A. Kutepov, S. Sharma, J. K. Dewhurst, E. K. U. Gross, A. I. Lichtenstein, and V. P. Antropov, Phys. Rev. B 96, 184418 (2017). 